\title{
ANALYSIS OF FATTY ACID COMPOSITION IN CHICKEN FAST FOODS OF DHAKA CITY
}

\author{
MAHMUDUR RAHMAN ${ }^{1 *}$, BIDHAN CHANDRA PAUL ${ }^{2}$, AYESHA SHARMIN ${ }^{2}$, MOHAMMAD \\ LOKMAN HOSSAIN ${ }^{1}$, SUBRATA CHANDRA ROY ${ }^{1}$, MALA KHAN ${ }^{3}$, MD JUWEL HOSEN ${ }^{3}$, \\ MD MAINUL HOSSAIN ${ }^{4}$
}

Department of Chemistry, Jagannath University, Dhaka-1100, Bangladesh

\begin{abstract}
Fatty acid composition in chicken fast food was analyzed by attenuated total reflectance-Fourier transform infrared (ATR-FTIR) spectroscopy and gas chromatography-flame ionization detector (GC-FID). Saturated fatty acids (SFA), monounsaturated fatty acids (MUFA) and polyunsaturated fatty acids (PUFA) have been found in various amounts in the fast food samples. None of the fast food samples contain trans fatty acid. Chicken Winglet (A) and Chicken Hot Wings (B) have higher amount of saturated fatty acids (SFA) which are $28.73 \%$ and $25.92 \%$ respectively. The amount of saturated fatty acids (SFA) in Chicken Drumst (C), Chicken Botik (D), Fiery Grilled Chicken (E), Chicken Meatballs (F), and Chicken Nuggets (G) are in between 10.94-19.38\%. The saturated fatty acids found in the fast food samples are palmitic acid, stearic acid, and myristic acid. Highest amount of linoleic acid (omega-6, 18.90\%) was found in Chicken Meatballs (F). The ratio of omega-6 and omega-3 in sample D was 2.32:1 which is in the acceptable range. Although trans fatty acid was not found in the samples, presence of more than $10 \%$ saturated fatty acids in chicken fast food is still harmful for health as it may increase risk of cardiovascular disease (CVD).
\end{abstract}

Keywords: chicken fast food, trans fatty acids (TFAs), saturated fatty acids (SFA), monounsaturated fatty acids (MUFA) and polyunsaturated fatty acids (PUFA).

\section{INTRODUCTION}

Fatty acids can be saturated or unsaturated. Unsaturated fatty acids can be monounsaturated fatty acids (MUFA) or polyunsaturated fatty acids (PUFA). Saturated fatty acid intake is known to increase low density lipoprotein (LDL) cholesterol, and therefore has been associated with increased risk of cardiovascular disease (CVD) (Siri-Tarino et al. 2010). Moreover, it is believed that human beings evolved on a diet with a ratio of omega- 6 to omega-3 essential fatty acids (EFA) of 1:1 (Simopoulos 2002, Simopoulos 2016 and Simopoulos 2008). Omega-6 to omega-3 fatty acid ratio of $5: 1$ or less is considered as less harmful for health (Rubio-Rodríguez et al. 2010). Now due to change in food habit and preference to fast food, people are having excessive amounts of omega- 6 fatty acids and less amount of omega- 3 in their food. This leads to increase omega- 6 to omega-3 ratio to 20:1 or even more (Simopoulos 2016). A high amount of omega- 6 polyunsaturated fatty acids (PUFA) in food and a high omega6/omega-3 ratio promote cardiovascular disease, cancer and inflammatory diseases (Simopoulos 2002). Recent studies show that omega-6 to omega-3 fatty acid ratio play important role in obesity (Simopoulos 2016).

Fast food makers sometimes prefer to use hydrogenated vegetable oil because this oil is inexpensive and have increased shelf life, remain stable during deep frying, and can improve the food's texture. Though the natural vegetable oils generally contain mostly unsaturated fat which is good for health, they are more prone to oxidation leading to the formation of hydroperoxides, and thus they more easily become rancid (Dixit et al. 2012). Dorni et al.

*Corresponding author: <mm_rahman1978@yahoo.co.uk>.

${ }^{1}$ Department of Chemistry, Jagannath University, Dhaka-1100, Bangladesh.

${ }^{2}$ Department of Chemistry, Bangladesh University of Engineering and Technology, Dhaka-1000, Bangladesh.

${ }^{3}$ Designated Reference Institute for Chemical Measurements, BCSIR, Dhaka-1205, Bangladesh.

${ }^{4}$ Department of Biochemistry and Microbiology, North South University, Dhaka-1229, Bangladesh. 
did a survey of fatty acid profile of edible oils and fats consumed in India (Dorni et al. 2018). They found vanaspati (also known as vegetable ghee) contains trans fatty acid 'elaidic acid' content, ranged from 1.04 to $12.09 \%$. Vanaspati is produced from vegetable oils using partial hydrogenation at a certain temperature and pressure in the presence of nickel as catalyst. This process modifies the fatty acid composition into saturation and isomerization of few cis double bonds to their trans forms by changing the position of hydrogen atom (Dorni et al. 2018). Trans fatty acids (TFAs) cause adverse effects on health. TFAs increase the risk of coronary heart disease, cancer and diabetes (Costa et al. 2016). Consumption of TFA increases the risk of type 2 diabetes (Bhardwaj et al. 2016). High intakes of saturated and trans fatty acids contribute to increased cardiovascular disease by lowering the 'good HDL cholesterol' and raising the 'bad LDL cholesterol' (Benatar et al. 2014, Menaa et al. 2013, Dixit et al. 2012, Downs et al. 2013, and Zevenbergen et al. 2009). Among various trans fatty acids, elaidic acid (C18:1 trans-9), and vaccenic acid (C18:1 trans-11) are considered mostly responsible for coronary heart disease (CHD) (Mozaffarian et al. 2009, and Katan (2006).

Some fast foods of Dhaka city have been investigated to know kinds of fatty acids present in it, the ratio of omega- 6 to omega- 3 in fast foods and to find the presence of any harmful trans fatty acids.

\section{MATERIALS}

For ATR-FTIR analysis, standard trans fatty acids such as palmitelaidic acid (C16:1, trans-9) and elaidic acid (C18:1, trans-9) were procured from Santa Cruz Biotechnology and transvaccenic acid (C18:1, trans-11) was purchased from Sigma-Aldrich. For GC-FID analysis, reference standard- Supelco 37 Component
FAME Mix (CRM47885) was purchased from Sigma Aldrich.

Chicken Winglet (A), Chicken Hot Wings (B) and Fiery Grilled Chicken (E) were collected from Dhanmondi, Dhaka. Chicken Drumst (C) and Chicken Botik (D) were collected from Mohammadpur, Dhaka. Chicken Meatballs (F) and Chicken Nuggets $(\mathrm{G})$ were collected from Mirpur, Dhaka.

Soxhlet extraction method was performed to extract fatty acids from the different samples. No cleanup procedure was performed as it may wash away fatty acids from the samples.

\section{METHODS}

Trans fatty acids (TFAs) were determined using attenuated total reflectance-Fourier transform infrared (ATR-FTIR) spectroscopy and gas chromatography-flame ionization detector (GCFID).

ATR-FTIR of liquid samples was performed in an ATR sample cell with a zinc selenide $(\mathrm{ZnSe})$ crystal from IRprestige-21 Shimadzu (Japan) Spectrometer. ZnSe crystal was mounted in the sample compartment of the spectrometer. All standards and samples were scanned over the range of $4000-650 \mathrm{~cm}^{-1}$ by 16 scans with a resolution of 4 $\mathrm{cm}^{-1}$. The ATR crystal was carefully cleaned by wiping with soft tissue paper and propanol to remove any lipophilic or hydrophilic residues from the previous sample. A fresh background spectrum of air was taken before recording the spectra of each standard and sample.

GC-FID was performed in a Shimadzu, GC2010 Plus (Tokyo, Japan) gas chromatograph fitted with a fused silica polar capillary column (75 m x $0.18 \mathrm{~mm}$ i.d $\times 0.14 \mu \mathrm{m}$ film thickness) coated with poly(biscyanopropyl siloxane) and flame ionization detector. GC-FID analysis was performed under following conditions: Initial column temperature was maintained at $180^{\circ} \mathrm{C}$ 
and held for $45 \mathrm{~min}$. Then the temperature was increased to a final temperature of $240^{\circ} \mathrm{C}$ at a rate of $4^{\circ} \mathrm{C} / \mathrm{min}$ and hold for $15 \mathrm{~min}$. Injector temperature: $250^{\circ} \mathrm{C}$, Injected volume: $1 \mu \mathrm{L}$, Injection mode: split, split ratio- 50:1. Detector temperature: $250^{\circ} \mathrm{C}$, column flow rate: 0.34 $\mathrm{mL} / \mathrm{min}$ and carrier gas: nitrogen.

\section{Extraction of fatty acids}

Samples were grinded as fine as possible without any pretreatment. Fatty acids in various food samples were extracted by the Soxhlet extraction method (Daugherty et al. 1983). $10 \mathrm{~g}$ of grinded food sample was accurately weighed and was put into a cellulose-extraction thimble, which was covered with cotton wool. $150 \mathrm{~mL}$ chloroform/methanol solvent $(2: 1, \mathrm{v} / \mathrm{v})$ was taken in a round bottom flask and used to extract fatty acids under reflux for 8 hours at $70^{\circ} \mathrm{C}$. Solvent was removed from the extract by using rotary evaporator and dried at $40-60^{\circ} \mathrm{C}$ for 1 hour.

Fatty acids were converted into fatty acid methyl esters (FAMEs) for GC-FID analysis. FAMEs were prepared according to method AOAC 969.33. To prepare FAME, lipids/ fats were hydrolyzed with methanolic $\mathrm{KOH}$ solution and the hydrolyzed lipids/ fats were transesterified by using $\mathrm{BF}_{3}$-methanol complex solution. Finally FAMEs were extracted with petroleum ether.

\section{RESULTS AND DISCUSSION}

Fatty acids have been extracted from fast foods using Soxhlet extraction method in chloroform/methanol solvent according to standard method (Daugherty et al. 1983). None of the samples were cleaned as it may wash away fatty acids from the samples. Attenuated total reflectance-Fourier transform infrared (ATR-FTIR) spectroscopy and gas chromatography-flame ionization detector (GCFID) have been used to determine trans fatty acids in the fast foods in Dhaka city.

\section{ATR-FTIR results}

In this study, elaidic acid (C18:1 trans-9), vaccenic acid (C18:1 trans-11) and palmitelaidic acid (C16: 1 trans-9) have been used as standard in ATR-FTIR analysis because elaidic acid (C18:1 trans-9) and vaccenic acid (C18:1 trans11) are considered as risk factor for coronary heart disease (CHD). The ATR-FTIR of sample A (Chicken Winglet) is shown in Fig. 1. ATRFTIR spectrum of three standard trans fatty acids and all fast food samples (A-G) are shown

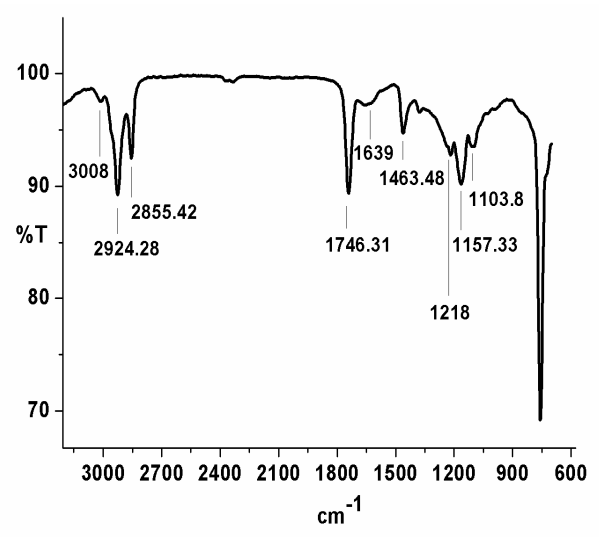

Fig. 1. ATR-FTIR of fatty acid from fast food sample A (Chicken Winglet).

in Fig. 2. Both Figs. 1 and 2 clearly show the presence of typical functional groups in fatty acids of the fast food samples. In Fig. 1, C-H stretching vibration of cis- double bond appears at $3008 \mathrm{~cm}^{-1}$. The band at $2924 \mathrm{~cm}^{-1}$ is due to asymmetric stretching of aliphatic methylene group, - $\mathrm{C}-\mathrm{H}\left(\mathrm{CH}_{2}\right)$ present in fatty acid of sample. The symmetric stretching of- $\mathrm{C}-\mathrm{H}\left(\mathrm{CH}_{2}\right)$ is at $2855 \mathrm{~cm}^{-1}$. The band at $1746 \mathrm{~cm}^{-1}$ is due to $-\mathrm{C}=\mathrm{O}$ stretching which arises from ester carbonyl group of triglyceride of fatty acid. The 
$\mathrm{C}=\mathrm{C}$ stretching vibration of cis-olefin appears at $1639 \mathrm{~cm}^{-1}$ as a very weak band. The $-\mathrm{C}-\mathrm{H}$ bending of the $\mathrm{CH}_{2}$ and $\mathrm{CH}_{3}$ of aliphatic groups appear at $1463 \mathrm{~cm}^{-1}$. The bands at $1218 \mathrm{~cm}^{-1}$, $1157 \mathrm{~cm}^{-1}$ and $1103 \mathrm{~cm}^{-1}$ are due to stretching vibration -C-O ester group (Vlachos et al. 2006).

An expanded ATR-FTIR spectrum of three standard trans fatty acids and fast food samples (A-G) are shown in Fig. 3 to show trans peak more clearly. Trans peak at $964 \mathrm{~cm}^{-1}$ is clearly found in all the three standard trans fatty acids namely elaidic acid, vaccenic acid, palmitelaidic acid (Fig. 2 and Fig. 3). However, none of the fast food samples (A-G) has any peak in the characteristic trans peak region from 976 to 956 $\mathrm{cm}^{-1}$. According to literature, trans peak is found in the region from $976-956 \mathrm{~cm}^{-1}$ with a maximum at $966 \mathrm{~cm}^{-1}$ which is due the $\mathrm{CH}$ outof-plane deformation absorption (Sherazi et al. 2009 and Juaneda et al. 2007).

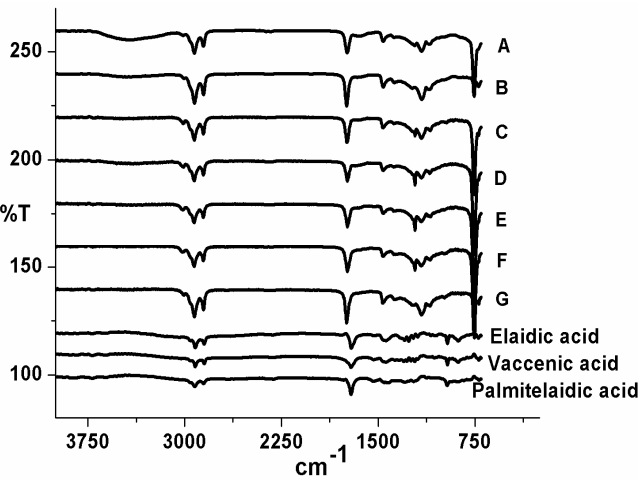

Fig. 2. ATR-FTIR of three standard trans fatty acids and fast food samples (A-G) from 700$4000 \mathrm{~cm}^{-1}$.

In ATR-FTIR (Fig. 2), C-H stretching vibration of cis-double bond appears at $3008 \mathrm{~cm}^{-1}$. The assymetric stretching of $\mathrm{CH}\left(-\mathrm{CH}_{2}\right)$ is at 2924 $\mathrm{cm}^{-1}$, symmetric stretching of $\mathrm{CH}\left(-\mathrm{CH}_{2}\right)$ is at $2855 \mathrm{~cm}^{-1}$. The ester group $(-\mathrm{C}=\mathrm{O})$ is observed at $1746 \mathrm{~cm}^{-1}$, bending of $-\mathrm{CH}\left(-\mathrm{CH}_{2}-, \mathrm{CH}_{3}\right)$ is at
$1463 \mathrm{~cm}^{-1}$ and stretching of $-\mathrm{C}-\mathrm{O}$ is at $1157 \mathrm{~cm}^{-1}$ of fatty acids present in the sample. The band at $1639 \mathrm{~cm}^{-1}$ is due to $\mathrm{C}=\mathrm{C}$ stretching vibration of cis-olefin.

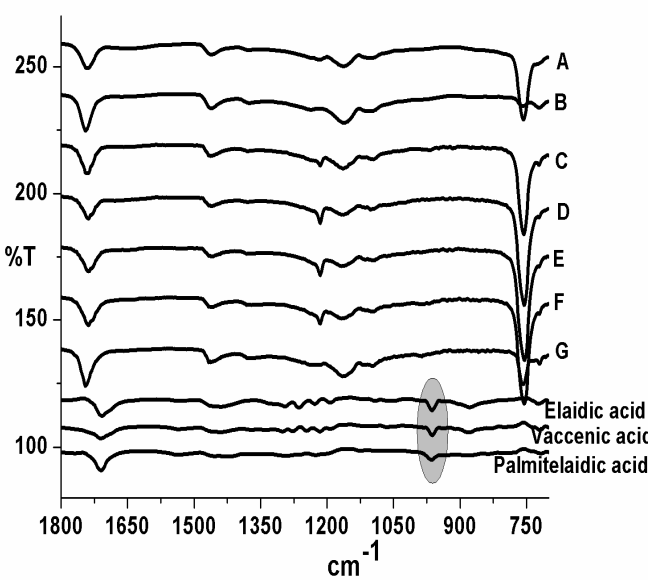

Fig. 3. ATR-FTIR (expanded) of three standard trans fatty acids and fast food samples (A-G) to show trans fatty acids from 976 to $956 \mathrm{~cm}^{-1}$ more clearly.

\section{GC-FID results}

ATR-FTIR provides information of trans fat containing more than 5\% (Sherazi et al. 2009). However, GC can give measurement at lower level of trans fat.

The results obtained from ATR-IR were further verified by the GC-FID. It is known that very long (around $100 \mathrm{~m}$ ) and highly polar capillary columns can provide better resolution of trans fatty acid isomers (Sherazi et al. 2009). For this reason, a polar capillary column (75 m long) coated with poly(biscyanopropyl siloxane) is used in GC analysis to maximize the resolution of trans fatty acid isomers. For GC-FID analysis, methyl esters of standards and samples were performed. Figs. 4 and 5 show the GC-FID chromatogram of standard fatty acids and sample D (Chicken Botik), respectively. Trans fatty acids were not found in any of the samples in GC-FID analysis (Fig. 5 and Table 1). 


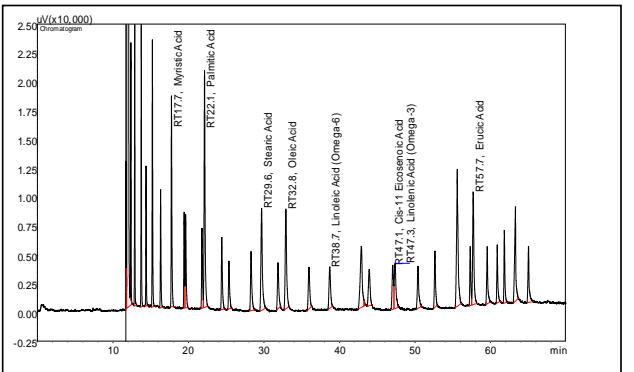

Fig. 4. GC-FID chromatogram of standard fatty acids

This result is consistent with the ATR-FTIR analysis. Table 1 shows the fatty acids and their contents that are present in each of the fast food samples by GC-FID analysis. Saturated fatty acids (SFA), monounsaturated fatty acids (MUFA) and polyunsaturated fatty acids (PUFA) have been found in the fast food samples. Chicken Winglet (sample A) and Chicken Hot Wings (sample B) have higher amount of saturated fatty acids (SFA) which are $28.73 \%$ and $25.92 \%$ respectively. The amount of saturated fatty acids (SFA) in Chicken Drumst, Chicken Botik, Fiery Grilled Chicken, Chicken Meatballs, and Chicken Nuggets are in between $10.94-19.38 \%$. Saturated fatty acids found in the fast food samples are palmitic acid, stearic acid, and myristic acid. All fast food samples contain oleic acid and linoleic acid (omega-6) in various

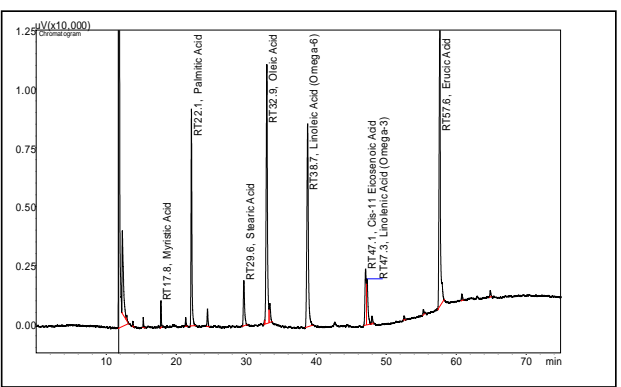

Fig. 5. GC-FID chromatogram of sample D (Chicken Botik).

amounts ranging from $12.47 \%$ to $28.53 \%$ and $8.30 \%$ to $18.90 \%$ respectively. Except sample D
(Chicken Botik) none of the sample contains linolenic acid (omega-3) fatty acid. In sample D, $8.30 \%$ linoleic acid (omega-6) and $3.57 \%$ linolenic acid (omega-3) are present. This shows omega- 6 to omega-3 ratio in sample D is $2.32: 1$ which is in the acceptable range.

Although fast food samples did not have any trans fatty acid, saturated fatty acids (SFA) content in each food is more than $10 \%$. The presence of higher amount of saturated fatty acids in the foods is still harmful as it can increase risk of cardiovascular disease (CVD). The World Health Organization (WHO) recommends for adults and children that saturated fatty acid intake should not be greater than $10 \%$ of total energy intake.

\section{CONCLUSION}

Saturated fatty acids (SFA), monounsaturated fatty acids (MUFA) and polyunsaturated fatty acids (PUFA) have been found in the fast food samples. None of the fast food samples that have been analyzed contain any trans fatty acids. The results of ATR-FTIR spectroscopy were found in good agreement with the results of GC-FID. All fast food samples contained omega-6 (linoleic acid) fatty acids in various amounts ranging from $8.30 \%$ to $18.90 \%$. The ratio of omega- 6 and omega-3 in Chicken Botik (D) was 2.32:1. This ratio is in the acceptable range. Except sample D, none of the fast food sample contains linolenic acid (omega-3). Chicken Winglet (A) and Chicken Hot Wings (B) have higher amount of saturated fatty acids which are $28.73 \%$ and $25.92 \%$, respectively. Intake of saturated fat is known to increase low density lipoprotein (LDL) cholesterol, and therefore has been associated with increased risk of cardiovascular disease (CVD).

\section{ACKNOWLEDGEMENT}

We thank University Grants Commission (UGC) of Bangladesh for financial support. 
Table 1: GC-FID analysis of fast food samples

\begin{tabular}{|c|c|c|c|c|}
\hline Sample ID & Type & Name of Acid & Unit & Amount (\%) \\
\hline \multirow{6}{*}{ A } & \multirow{3}{*}{ SFA } & Palmitic Acid & $\%$ & 20.64 \\
\hline & & Stearic Acid & $\%$ & 6.07 \\
\hline & & Myristic Acid & $\%$ & 2.02 \\
\hline & MUFA & Oleic Acid & $\%$ & 12.47 \\
\hline & PUFA & Linoleic Acid (Omega-6) & $\%$ & 13.49 \\
\hline & & & Total $=$ & 54.69 \\
\hline \multirow{6}{*}{ B } & \multirow[t]{2}{*}{ SFA } & Palmitic Acid & $\%$ & 24.33 \\
\hline & & Stearic Acid & $\%$ & 1.59 \\
\hline & \multirow[t]{2}{*}{ MUFA } & Oleic Acid & $\%$ & 28.53 \\
\hline & & Palmitoleic Acid & $\%$ & 1.19 \\
\hline & PUFA & Linoleic Acid (Omega-6) & $\%$ & 8.53 \\
\hline & & & Total $=$ & 64.17 \\
\hline \multirow{6}{*}{$\mathrm{C}$} & \multirow[t]{2}{*}{ SFA } & Palmitic Acid & $\%$ & 10.93 \\
\hline & & Stearic Acid & $\%$ & 1.78 \\
\hline & \multirow[t]{2}{*}{ MUFA } & Oleic Acid & $\%$ & 19.89 \\
\hline & & Palmitoleic Acid & $\%$ & trace \\
\hline & PUFA & Linoleic Acid (Omega-6) & $\%$ & 17.20 \\
\hline & & & Total= & 49.80 \\
\hline \multirow{9}{*}{$\mathrm{D}$} & \multirow{3}{*}{ SFA } & Palmitic Acid & $\%$ & 8.92 \\
\hline & & Stearic Acid & $\%$ & 1.04 \\
\hline & & Myristic Acid & $\%$ & trace \\
\hline & \multirow[t]{3}{*}{ MUFA } & Oleic Acid & $\%$ & 17.85 \\
\hline & & Erucic Acid & $\%$ & 32.67 \\
\hline & & cis-11 Eicosenoic Acid & $\%$ & 4.46 \\
\hline & \multirow{3}{*}{ PUFA } & Linoleic Acid (Omega-6) & $\%$ & 8.30 \\
\hline & & Linolenic Acid (Omega-3) & $\%$ & 3.57 \\
\hline & & & Total= & 76.81 \\
\hline \multirow{7}{*}{$\mathrm{E}$} & \multirow{2}{*}{ SFA } & Palmitic Acid & $\%$ & 8.75 \\
\hline & & Stearic Acid & $\%$ & 3.56 \\
\hline & \multirow[t]{3}{*}{ MUFA } & Oleic Acid & $\%$ & 13.63 \\
\hline & & Palmitoleic Acid & $\%$ & 1.75 \\
\hline & & cis-11 Eicosenoic Acid & $\%$ & trace \\
\hline & \multirow[t]{2}{*}{ PUFA } & Linoleic Acid (Omega-6) & $\%$ & 9.43 \\
\hline & & & Total= & 37.12 \\
\hline \multirow{8}{*}{$\mathrm{F}$} & \multirow{3}{*}{ SFA } & Palmitic Acid & & \\
\hline & & $\begin{array}{c}\text { Palmitic Acid } \\
\text { Stearic Acid }\end{array}$ & $\begin{array}{l}\% \\
\%\end{array}$ & $\begin{array}{c}16.64 \\
1.40\end{array}$ \\
\hline & & Myristic Acid & $\%$ & 1.34 \\
\hline & \multirow[t]{3}{*}{ MUFA } & Oleic Acid & $\%$ & 18.29 \\
\hline & & Palmitoleic Acid & $\%$ & 1.03 \\
\hline & & cis-11 Eicosenoic Acid & $\%$ & 1.41 \\
\hline & \multirow[b]{2}{*}{ PUFA } & Linoleic Acid (Omega-6) & $\%$ & 18.90 \\
\hline & & & Total= & 59.01 \\
\hline \multirow{7}{*}{ G } & \multirow[t]{2}{*}{ SFA } & Palmitic Acid & $\%$ & 13.33 \\
\hline & & Stearic Acid & $\%$ & 1.5 \\
\hline & MUFA & Oleic Acid & $\%$ & 21.66 \\
\hline & & Palmitoleic Acid & $\%$ & 3.00 \\
\hline & & cis-11 Eicosenoic Acid & $\%$ & 1.41 \\
\hline & PUFA & Linoleic Acid (Omega-6) & $\%$ & 16.66 \\
\hline & & & To & $\mathbf{5 7 . 5 6}$ \\
\hline
\end{tabular}




\section{REFERENCES}

Bhardwaj, S., S. J. Passi, A. Misra, K. K. Pant, K. Anwar, R. M. Pandey and V. Kardam. 2016. Effect of heating/ reheating of fats/oils, as used by Asian Indians, on trans fatty acid formation. Food Chem. 212: 663-670.

Benatar, J. R., and R. A. Stewart. 2014. The effects of changing dairy intake on trans and saturated fatty acid levels- results from a randomized controlled study. Nutr. J. 13:32-42.

Costa, N., R. Cruz, P. Graca, J. Breda and S. Casal. 2016. Trans fatty acids in the Portuguese food market. Food Cont. 64: 128-134.

Dixit, S., and M. Das. J. 2012. Fatty acid composition including trans-fatty acids in edible oils and fats: probable intake in Indian population. Food Sci. 77:188-199.

Downs, S. M., A. M. Thow, S. Gosh-Jerath, J. McNab, K. S. Reddy, and S. R. Leeder. 2013. From Denmark to Delhi: the multisectoral challenge of regulating trans fats in India. Public Health Nutr. 16: 2273-2280.

Dorni, C., P. Sharma, G. Saikia, and T. Longvah. 2018. Fatty acid profile of edible oils and fats consumed in India. Food Chem. 238: 9-15.

Daugherty, CE., and H. G. Lento. 1983. Chloroform-methanol extraction method for determination of fat in foods: collaborative study. J. AOAC Int. 66: 927-932.

Juaneda, P., M. Ledoux, and J. L. Sebedio. 2007. Analytical methods for determination of trans fatty acid content in food. Eur. J. Lipid Sci. Technol. 109: 901-917.

Katan, M. B. 2006. Regulation of trans fats: The gap, the Polder, and McDonald's French fries. Atherosclerosis Supp. 7: 63-66.

Khan, M. U., M. F. Hasan, and A. Rauf. 2017. Determination of Trans Fat in Selected Fast Food Products and Hydrogenated Fats of India Using Attenuated Total Reflection Fourier Transform Infrared (ATR-FTIR) Spectroscopy. J. Oleo. Sci. 66: 251-257.
Menaa F., A. Menaa, B. Menaa, J. Treton. 2013. Trans-fatty acids, dangerous bonds for health? A background review paper of their use, consumption, health implications and regulation in France. Eur. J. Nutr. 52: 1289-1302.

Mozaffarian, D., A. Aro and W. C. Willett. 2009. Health effects of trans-fatty acids: experimental and observational evidence. Eur. J. Clin. Nutr. 63: $5-21$.

Rubio-Rodríguez, N., S. Beltrán , I. Jaime, S. M. de Diego, M.T. Sanz, and J. R. Carballido. 2010. Production of omega-3 polyunsaturated fatty acid concentrates: A review. Innov. Food Sci. Emerg. Technol. 11: 1-12.

Sherazi, S. T. H., A. Kandhro, S. A. Mahesar, M. I. Bhanger, M. Y. Talpur, and S. Arain. 2009. Application of transmission FT-IR spectroscopy for the trans fat determination in the industrially processed edible oils. Food Chem. 114: 323-327.

Siri-Tarino, P. W., Q. Sun, F. B. Hu, and R. M. Krauss. 2010. Saturated fatty acids and risk of coronary heart disease: modulation by replacement nutrients. Curr. Atheroscler. Rep. 12: $384-390$

Simopoulos, A. P. 2016. An increase in the omega6/omega-3 fatty acid ratio increases the risk for Obesity. Nutrients. 8: 128-137.

Simopoulos, A. P. 2008. The importance of the omega-6/omega-3 Fatty Acid ratio in cardiovascular disease and other chronic diseases. Exp. Biol. Med. 233: 674-688.

Simopoulos, A.P. 2002. The importance of the ratio of omega-6/omega-3 essential fatty acids. Biomed Pharmacother. 56: 365-379.

Vlachos, N., Y. Skopelitis, M. Psaroudaki, V. Konstantinidou, A. Chatzilazarou, and E. Tegou, 2006. Applications of Fourier transform-infrared spectroscopy to edible oils. Anal. Chim. Acta. 573-574: 459-465.

Zevenbergen, H., A. De. Bree, M. Zeelenberg, K. Laitinen, G. Van Duijn, and E. Floter. 2009. Foods with a high fat quality are essential for healthy diets. Ann. Nutr. Metab. 54: 15-24.

(Received revised manuscript on 01 April 2019) 